\title{
Aetiology and outcome in 53 cases of native valve staphylococcal endocarditis
}

\author{
V Hricak, J Kovacik, P Marks, D West, V Kromery Jr
} Cardiovascular Disease, Bratislava V Hricak

Clinic of Infectious Disease, Derer's Hospital, Bratislava J Kovacik

North London

Postgraduate Medical Centre, London UK

P Marks

Department of Health Management/Statistics, University of Scranton, Scranton, PA, USA

D West

V Kromery Jr

Department of Medicine, University of Trnava, Trnava,

Slovak Republic

V Kromery Jr

Correspondence to Dr Peter Marks, 1 Maxted Road, London SE15 4LL, UK

Accepted 20 April 1999
National Institution of

\begin{abstract}
Summary
Within the last 30 years the profile of infective endocarditis has altered considerably with regard to microbiological causation, clinical features, and natural history. A contributory factor has undoubtedly been the development of potent antibiotics and their sometimes indiscriminate use. The increase in intravenous drug abuse in urban centres, the use of immunosuppressive agents, and the use of prosthetic heart valves have also all contributed. Although cardiac surgery in the uninfected heart provides a perfect environment for infective endocarditis, the improved design of prosthetic valves and the enhanced long-term survival and decreased immediate operative risk, means that surgery is viewed as the best option in many cases. In a series of 53 cases of staphylococcal endocarditis from a national endocarditis survey, those risk factors which influenced outcome were analysed. Thirty out of 53 patients had predisposing heart disease. Mortality was $39.6 \%$. Statistical analysis revealed that attributable mortality was significantly associated with skin infection, systemic embolisation, and inappropriate therapy. Interestingly, surgical treatment was associated with better outcome.
\end{abstract}

Keywords: Staphylococcus aureus; endocarditis; cardiac surgery

The epidemiology of endocarditis is remarkable for its constancy. Up to the 1950s it was linked to the occurrence of rheumatic heart disease. Native valve disease could affect the damaged mitral or sometimes the aortic valve.

Table 1 National surveillance of endocarditis and incidence of staphylococcal endocarditis

\begin{tabular}{lllll}
\hline & \multicolumn{5}{l}{ Caused by } \\
\cline { 3 - 5 } Country & $\begin{array}{l}\text { No of } \\
\text { cases }\end{array}$ & $\begin{array}{l}\text { Staphylococci } \\
(\%)\end{array}$ & $\begin{array}{l}\text { Saureus } \\
(\%)\end{array}$ & $\begin{array}{l}\text { Coagulase-negative } \\
\text { staphylococci (\%) }\end{array}$ \\
\hline Argentina 1988-94 & 39 & 21.0 & $\mathrm{NA}$ & $\mathrm{NA}$ \\
Spain ${ }^{\star}$ 1978-93 & 1529 & 77.8 & 74.0 & 3.8 \\
France 1990-91 & 423 & 24.5 & 21.1 & 3.3 \\
Australia 1988-93 & 90 & 40.0 & $\mathrm{NA}$ & $\mathrm{NA}$ \\
Argentina 1990-94 & 285 & 32.0 & 24.5 & 7.5 \\
Slovakia 1990-96 & 180 & 35.0 & 23.0 & 12.0 \\
Israel 1980-94 & 83 & 11.4 & 4.3 & 7.1 \\
Greece 1989-93 & 131 & 19.8 & $\mathrm{NA}$ & $\mathrm{NA}$ \\
\hline
\end{tabular}

^Intravenous drug users; NA: not available
Bacteraemia from the teeth was a common origin, since dental caries made this likely and it has been established that invasive dental procedures produce transient bacteraemia. ${ }^{12}$ During the mid-1940s, however, clinical investigators discovered that this insidious malady could be treated with penicillin or prevented with prophylactic doses of the drug prior to dental procedures. The reduced incidence of rheumatic fever since the mid-1940s, with a concomitant reduction in heart valve damage, combined with the use of penicillin, has lowered the number of cases. Coincidental with the decrease in rheumatic heart disease and improvements in dental hygiene has been the increase in other origins of infection, such as intravenous drug abuse, central or peripheral intravenous catheters, cardiac surgery, and dialysis. ${ }^{3}$ Many of these origins of infection predispose to staphylococcal aetiology.

Oral strains of Streptococcus viridans were responsible for over $70 \%$ of cases of infective endocarditis in the pre-antibiotic era. They now constitute less than half such cases. Prior to 1990 , staphylococci represented only $10-$ $15 \%$ of all cases. However, within the last 7 years, they have increased to at least $23 \%$ of all cases and in some reports as many as $40 \% .{ }^{4}{ }^{5} \mathrm{~A}$ national survey in Slovakia found that staphylococci accounted for 33\% of all cases, and they were the most frequent pathogen. Streptococcus viridans accounted for $25 \%$, HACEK group accounted for $15 \%$, and enterococci for $12.5 \% .^{6}$ Staphylococcus aureus was the most common staphylococcal pathogen. ${ }^{7}$ In the context of European infection (table 1), several national surveys showed an incidence of staphylococcal endocarditis ranging from $11 \%$ to $40 \% .^{78101214}$ The purpose of this study was to analyse prospectively causation, treatment results, and risk factors in cases of staphylococcal endocarditis which presented within the last 7 years in the Slovak Republic.

\section{Patients and methods}

Between 1990 and 1996 all cases of native valve infective endocarditis of staphylococcal origin were prospectively evaluated using a protocol which was submitted to all medical departments in the Slovak Republic. No cases of prosthetic endocarditis were included.

Risk factors were recorded. These were underlying heart disease, rheumatic heart disease, source of bacteraemia, invasive procedures (catheterisation, endoscopy, drug abuse, dialysis, and prior surgery), other skin, soft tis- 
sue or catheter infection, or neoplasia. Major and minor criteria of the Duke Endocarditis service ${ }^{15}$ were recorded for definition of infective endocarditis as definite or possible.

The outcome of therapy was recorded as well as the antibiotics used and whether cardiac surgery was required. Aetiology of endocarditis was determined by positive blood culture from a peripheral vein. The Bactec (BectonDickinson BBL Laboratories, US) system for blood culture, and Vitek Jr (Vitek Systems, US) or API (Bio Merieux, Hazelwood, US) systems for identification of staphylococci were used.

DEFINITIONS

Definite or probable endocarditis was defined according to Duke criteria. ${ }^{15}$ There were 48 cases of definite $(90.6 \%)$ and 5 cases of probable native valve endocarditis. Previous surgery was defined as cardiosurgical procedure other than valvular replacement (only native valve endocarditis was considered in this study). Staphylococcal endocarditis was defined as endocarditis plus isolation of staphylococci from blood cultures. Two positive cultures of $S$ aureus or three cultures of coagulase-negative staphylococci from native value (biopsy, autopsy) were necessary for a definite diagnosis of staphylococcal endocarditis. Isolation of less than two positive cultures of $S$ aureus or three cultures of coagulase-negative staphylococci from blood cultures were considered as probable endocarditis.

Appropriate therapy was defined as administration of a combination of two antistaphylococcal antibiotics for at least 28 days in the absence of cardiosurgical valve replacement. In endocarditis, when surgical therapy (valve replacement) was done, 14 days of subsequent antibiotic therapy with a combination of anti-staphylococcal agents was considered appropriate therapy. Shorter therapy, or singleagent therapy, was considered inappropriate therapy except in cases where cardiosurgery was performed.

Attributable mortality was defined as patient death within 28 days of onset of infective endocarditis, or septic shock, or complications of endocarditis. Deaths for other reason within 28 days were assessed as crude mortality. There were no deaths after 28 days from the first positive blood culture.

Indications for surgery were the absence of clinical response after 5 days of antibiotic therapy with in vitro active antibiotic and repeated embolisation.

\section{STATISTICAL ANALYSIS}

The subgroup of staphylococcal endocarditis with a fatal outcome was compared with the subgroup of patients that survived, to determine risk factors for death in univariate analysis. The $\chi^{2}$ test was used in this univariate analysis for significance of risk factors for outcome and a p-value of less than 0.05 was considered to be significant.
Aetiology, risk factors and outcome of 53 cases of staphylococcal endocarditis

Major criteria:

- typical bacteria in blood (A) or $>3$ positive cultures (B): 41

- positive echocardiography: 36

Minor criteria:

- predisposing heart condition: 41

- fever $>38^{\circ} \mathrm{C}: 52$

- vascular phenomena: 31

- immunologic phenomena: 39

- minor echocardiographic changes: 5

- positive blood culture, other than (A) or (B): 14

Duke classification: 48 definite; 5 possible

Localisation:

- aortic: 22

- mitral: 25

- right: 6

Outcome:

- surgery: 11

- survival: 32

- death: 21

\section{Results}

There were 53 cases of subacute bacterial endocarditis within the 7-year period studied. The patient characteristics, risk factors, and results of treatment are listed in the box. Attributable mortality was $39.6 \% ; 32$ patients survived and 21 died. In $75.5 \%$ of cases, $S$ aureus was the causative organism and in $24.5 \%$ it was coagulase-negative staphylococci ( $S$ epidemidis, $S$ warnei, and $S$ xylosus). An analysis of risk factors revealed that rheumatic fever was the most common underlying disease, accounting for $39.6 \%$ of cases; this was followed by congenital cardiac abnormalities, accounting for $13.2 \%$.

Interestingly, only a minority of endocarditis patients had what are considered some of the typical risk factors such as invasive procedures, eg, catheterisation, endoscopy, or dialysis $(3.8 \%, 3.8 \%$, and $5.7 \%$, respectively). Only 3-8\% were drug abusers, no doubt because of the low incidence of drug abuse in Slovakia. Seventeen out of 53 patients with endocarditis $(32.1 \%)$ were over 60 years old. Using the Duke criteria, ${ }^{15} 90.6 \%$ were definite and $9.4 \%$ probable. In a surprisingly high proportion of cases, infective endocarditis was difficult to diagnose with certainty. This is due both to the inaccessibility of the intracardicac vegetations, and the highly variable and sometimes non-specific nature of the clinical manifestations.

In 1981 Von Reyn and colleagues ${ }^{16}$ published a paper entitled 'Infective endocarditis: an analysis based upon strict case definitions'. However, the Von Reyn criteria, especially their failure to utilise echocardiographic findings, have been largely replaced by the Duke endocarditis service criteria for diagnosis of infective endocarditis, with its utilisation of echocardiographic findings. Echocardiography is now routinely used in evaluating patients 
Table 2 Predisposing conditions, treatment and results

\begin{tabular}{|c|c|c|c|c|}
\hline & $\begin{array}{l}\text { Total } \\
(n=53)\end{array}$ & $\begin{array}{l}\text { Survivors } \\
(n=32)\end{array}$ & $\begin{array}{l}\text { Death } \\
(n=21)\end{array}$ & $p<$ \\
\hline \multicolumn{5}{|l|}{ Predisposing conditions } \\
\hline Rheumatic fever & $21(39.6)$ & $14(43.8)$ & $7(33.3)$ & NS \\
\hline Congenital heart disease & $7(13.2)$ & $2(6.4)$ & $5(23.9)$ & NS \\
\hline Other cardiologic disease & $2(3.8)$ & $1(3.2)$ & $1(4.8)$ & NS \\
\hline Neoplasia & $3(5.7)$ & $2(6.3)$ & $1(4.8)$ & NS \\
\hline \multicolumn{5}{|l|}{ Procedures } \\
\hline Drug abuse & $2(3.8)$ & $1(3.2)$ & $1(4.8)$ & NS \\
\hline Central catheterization & $2(3.8)$ & $1(3.1)$ & $1(4.8)$ & NS \\
\hline Endoscopy & $2(3.8)$ & $1(3.1)$ & $1(4.8)$ & NS \\
\hline Dialysis & $3(5.7)$ & $1(3.1)$ & $2(9.6)$ & NS \\
\hline Cardiosurgery (other valve replacement) & $7(13.2)$ & $4(12.5)$ & $3(14.3)$ & NS \\
\hline Dental surgery & $4(7.6)$ & $2(6.3)$ & $2(9.6)$ & NS \\
\hline Other surgery & $2(3.8)$ & $1(3.1)$ & $1(4.8)$ & NS \\
\hline \multicolumn{5}{|l|}{ Aetiology } \\
\hline Unknown & $35(66.0)$ & $19(59.4)$ & $7(33.3)$ & NS \\
\hline Other & $5(9.4)$ & $3(9.4)$ & $2(9.6)$ & NS \\
\hline Skin & $11(20.8)$ & $8(25.0)$ & $11(52.4)$ & NS \\
\hline Suspected site of infection/colonization & $3(50.7)$ & $2(6.3)$ & $1(4.8)$ & NS \\
\hline Nosocomial infective endocarditis & $6(11.3)$ & $3(9.4)$ & $3(14.3)$ & NS \\
\hline Tonsillitis & $6(11.3)$ & $3(9.4)$ & $3(14.3)$ & NS \\
\hline Age over 60 & $17(32.1)$ & $8(25.0)$ & $9(42.8)$ & NS \\
\hline Skin of soft tissue infection & $2(3.8)$ & $1(3.1)$ & $1(4.8)$ & NS \\
\hline Diabetes mellitus on insulin & $6(11.3)$ & $3(9.4)$ & $3(13.2)$ & NS \\
\hline \multicolumn{5}{|l|}{ Other predisposing conditions } \\
\hline $\begin{array}{l}\text { Infection with coagulase-negative } \\
\text { staphylococci }{ }^{\star}\end{array}$ & $13(24.5)$ & $8(25.0)$ & $5(23.9)$ & NS \\
\hline Infection with $S$ aureus & $40(75.5)$ & $27(78.1)$ & $15(71.4)$ & NS \\
\hline \multicolumn{5}{|l|}{ Definitions and therapy } \\
\hline Duke definite & $48(90.6)$ & $28(87.5)$ & $20(95.2)$ & NS \\
\hline Duke possible & $5(9.4)$ & $4(12.5)$ & $1(4.8)$ & NS \\
\hline \multicolumn{5}{|l|}{ Localisation: } \\
\hline - aortic & $22(41.6)$ & $10(31.2)$ & $12(57.2)$ & NS \\
\hline - mitral & $20(37.7)$ & $14(43.8)$ & $6(28.5)$ & NS \\
\hline - tricuspid/pulmonary & $7(13.2)$ & $5(15.6)$ & $2(9.6)$ & NS \\
\hline Embolisation & $31(58.5)$ & $14(43.8)$ & $17(80.9)$ & 0.02 \\
\hline Appropriate therapy: total & $29(54.8)$ & $23(71.9)$ & $6(28.7)$ & 0.005 \\
\hline - medical only & $18(34.0)$ & $13(40.7)$ & $5(23.9)$ & NS \\
\hline - surgery plus antibiotics & $11(20.8)$ & $10(31.2)$ & $1(4.8)$ & 0.04 \\
\hline Inappropriate therapy: total & $24(45.3)$ & 7 (21.9) & $17(80.1)$ & 0.001 \\
\hline - wrong antibiotics (combination) & $18(34.0)$ & $6(18.8)$ & $12(57.2)$ & 0.01 \\
\hline - short therapy & $6(11.3)$ & $1(3.1)$ & $5(23.9)$ & 0.03 \\
\hline
\end{tabular}

${ }^{\star} S$ warneri (2), S xylosus (1), S epidermidis (10), NS: non-significant

with suspected endocarditis, reflecting the improved capability of this technique to detect vegetations. This development alone provides sufficient justification for a major revision of the diagnostic criteria of endocarditis.

The most common site of the infection was the aortic valve followed by the mitral valve in $37.7 \%$ of cases. Valves on the right side were involved in $13.2 \%$ of cases.

According to the definitions, appropriate therapy was administered to only $54.3 \%$ of patients.

Two subgroups of patients were compared. The purpose of the comparison was to investigate specific risk factors for mortality in the patients who survived ( 32 patients) compared with those who died (21 patients). Embolisation was a major risk factor, being present in $80.9 \%$ of the group who died compared with $43.8 \%$ of those who survived $(\mathrm{p}<0.02)$. Inappropriate therapy was a major risk factor, being present in $80.1 \%$ of those who died compared with $21.9 \%$ of those who survived $(p<0.001)$. Therapy with too short a course was significant $(\mathrm{p}<0.03)$, as was treatment with the wrong antibiotics or without combination therapy $(\mathrm{p}<0.01)$. Skin infection as a possible origin of endocarditis was also found to be statistically significant $(p<0.005)$ and these results are shown in table 2 .

\section{Discussion}

Effective antibiotic therapy has favourably influenced the natural history of infective endocarditis. The disease was once fatal; now survival rates of more than $50 \%$ are achieved. Cardiac valve replacement has further improved survival. However, despite improvements, mortality remains near $30 \%$. Mortality was higher in our group (39.6\%) than in other studies (25-33\%), probably because of the high percentage $(45.3 \%)$ of inappropriate therapy. The analysis of typical risk factors yielded several noteworthy results. Only two patients were intravenous drug addicts, unlike figures from studies carried out in Western Europe and the US, where this was a major risk factor. The different profile of drug abuse and HIV infection in Slovakia is the most probable explanation. Within the 7 years of the study there were about 3000 intravenous drug abusers, 74 HIV-positive patients, and only seven AIDS-related deaths. With regard to aetiology, $75.5 \%$ of infections were caused by $S$ aureus.

The analysis of the 10-year treatment of infective endocarditis at the University of Alabama Medical Centre recommended early surgery for patients with native valve endocarditis and moderate or severe heart failure, and for all patients with staphylococcal native valve endocarditis, regardless of haemodynamic state. They stress that staphylococcal endocarditis was associated with a mortality of $44 \%$ treated non-surgically but, more importantly, was associated with multiple complicating events. They mention annular and myocardial abscesses and also coronary emboli and heart block. ${ }^{17}$ In our series, patients treated surgically had significantly lower mortality than those treated medically with appropriate therapy (4.8 vs $23.9 \%, \mathrm{p}<0.04)$. It must also be said that there was an unexpectedly high percentage of inappropriate therapies $(45.3 \%)$ which were not surprisingly associated with a worse outcome; $28.7 \%$ of those treated appropriately died, compared with $80.1 \%$ of those treated inappropriately.

In a surprisingly high proportion of cases, infective endocarditis is difficult to diagnose with certainty. This is due both to the inaccessibility of intracardiac vegetations and the highly variable and sometimes non-specific nature of the clinical manifestations. Over- and under-diagnosis of infective endocarditis are both still common. The changing clinical picture of endocarditis has made its clinical diagnosis even more difficult. The classical pattern of subacute bacterial endocarditis characterised by a protracted history of constitutional symptoms is infrequently seen today and has been replaced by an acutely toxic and more febrile picture. Whereas oral Streptococcus viridans was responsible for over $70 \%$ of infective endocarditis in the pre-antibiotic era, most of the cases seen today are due to staphylococci. ${ }^{18}$ These difficulties in making a definite diagnosis account for the development of the Duke criteria for the diagnosis of infective endocarditis, superceding the Von Reyn criteria. Inappropriate therapy is an 
unfortunate consequence of inaccurate diagnosis which again is due to the variable nature and manifestations of the disease.

Whilst infective endocarditis is a disease which is firmly part of the history of medicine, it is also sufficiently current to warrant further

1 Chiodo G, Tolle SW, Bartley M. Antibiotic prophylaxis for dental treatment. Illinois Dent f 1990:599-602.

2 Adams DP. Subacute bacterial endocarditis and dental practice: a brief survey, 1900-1950. Bull Hist Dent 1990;38(2):25-8.

3 Bayer AS, Ward JI, Ginzton LE, Shapiro SM. Evaluation of new classification criteria for the development of infective new classification criteria for the devel

4 Wilson APR, Gaya U. Treatment of endocarditis with teicoplanin. $\mathcal{F}$ Antimicrob Chemother 1996;38:507-21

5 Delahaye F, Goulet V, Lacassin F. Characteristics of infective endocarditis in France in 1991: a 1 year survey. Eur infective endocarditis in F

6 Hricak V, Kovacik J, Krcmery V. Infective endocarditis in Slovakia. A national survey. Acta Chemother 1997;6:33-7.

7 Riesenberg U, Urich N, Porath A, Schlaeffer F. Infective endocarditis in a community hospital in a fifteen year period (Abstract). Abstracts of the $3^{\text {rd }}$ International Symposium on Modern Concepts of Endocarditis. Boston, USA, 13-15 July 1995; No 134

8 Miro JM, Crucetta JM. Infective endocarditis in Spanish iv drug addicts: analysis of 1529 episodes (Abstract). Abstracts of the $3^{\text {rd }}$ International Symposium on Modern Concepts of Endocarditis. Boston, USA, 13-15 July 1995; No 109.

9 Bologna, R, Stamboulian, D. Infective endocarditis in a $3^{\text {rd }}$ International Symposium on Modern Concepts of Endocarditis. Boston, USA, 13-15 July 1995; 147.

10 Leport C, Lacassin F, Etienne J, et al. Infective endocarditis due to Staphylococcus spp. Data from a one year nation due to Staphylococcus spp. Data from a one year nation
wide survey (Abstract). Abstracts of the $3^{\text {rd }}$ International wide survey (Abstract). Abstracts of the $3^{\text {Id }}$ International
Symposium on Modern Concepts of Endocarditis. Boston, Symposium on Modern Concep. analysis of its diagnosis and treatment. New routes of infection and improved diagnostic techniques call for a fresh appraisal of management and treatment.

11 Kardavas FG, Savoglon G, Koliopoulos LP, Lolas C. Ten years surveillance of infective endocarditis in a large referral hospital in Greece (Abstract). Abstracts of the $3^{\text {rd }}$ International Symposium on Modern Concepts of Endocarditis. Boston, USA, 13-15 July 1995; No 144.

12 Hale GE, Spelman D. Infective endocarditis at the Alfred Hospital (Abstract). Abstracts of the $3^{\text {rd }}$ International Symposium on Modern Concepts of Endocarditis. Boston, USA, 13-15 July 1995; No 145 .

13 Arguello E, and the Study Group of Argentinian Society of Cardiology. Infective endocarditis in the Argentine Republic (Abstract). Abstracts of the $3^{\text {rd }}$ International Symposium on Modern Concepts of Endocarditis. Boston, USA, 13-15 July 1995; No 152a.

14 Hricak V, Matejika F, Sedlak T, et al. Native valve staphylococcal endocarditis: etiology, risk factors and outcome in 53 cases. F Chemother 1998;10:416-9.

15 Durack DT, Lukes AS, Bright DK. The Duke Endocarditis Service criteria for diagnosis of infective endocarditis: utilisation of echocardiographic findings. Am f Med 1994;96: sation

16 Von Reyn CF, Levy BS, Arbeit RD, Friedland G, Crumpacker CS. Infective endocarditis: an analysis based on strict case definitions. Ann Interm Med 1981;94:505-17.

17 Richardson JV, Karp RB, Kirklin JW, Dismukes WE. Treatment of infective endocarditis: a 10 year comparative analysis. Circulation 1978;4:589-97.

18 Rapport E. The changing role of surgery in the management of infective endocarditis (Editorial). Circulation 1978; $4: 598-9$

Ivan Petrovitch Pavlov (1849-1936) was born in the Riazan district of Russia, son of a priest, and eventually become the greatest experimental physiologist of his day. He graduated as MD in St Petersburg (1879) and became professor of pharmacology at the Military Academy and physician to the Medico-Chirurgical Academy. The Pavlov reflex is now an everyday household word but it guided him to the Nobel Prize and Fellowship of the Royal Society (1915) and to great acclamation when he attended the International Neurological Congress in London (1935). He died on 27 February 1936. - DG Fames 